\title{
Spatial Neglect Predicts Upper Limb Use in the Activities of Daily Living
}

\author{
Tim Vanbellingen ${ }^{\mathrm{a}-\mathrm{c}}$ Beatrice Ottiger ${ }^{c}$ Noortje Maaijwee ${ }^{c}$ \\ Tobias Pflugshaupt ${ }^{c}$ Stephan Bohlhalter ${ }^{c}$ René M. Müria ${ }^{a, b}$ \\ Tobias Nef ${ }^{a}$ Dario Cazzoli ${ }^{a}$ Thomas Nyffeler ${ }^{a-c}$
}

${ }^{a}$ Gerontechnology and Rehabilitation Group, and ${ }^{b}$ Perception and Eye Movement Laboratory, Department of Neurology, University of Bern, Bern, and ${ }^{\mathrm{C}}$ Neurocenter, Luzerner Kantonsspital, Lucerne, Switzerland

\section{Keywords \\ Spatial neglect - Functional outcome - Activities of daily living $\cdot$ Upper limb $\cdot$ Stroke $\cdot$ Right hemisphere}

\begin{abstract}
Background and Purpose: Motor tests performed at stroke onset have been shown to predict the recovery of upper limb motor impairment. Less is known about upper limb recovery at the level of functional activity or of participation and how spatial neglect may influence the integration of the upper limb in the activities of daily living (ADL). Our objective was to investigate whether the initial severity of spatial neglect may predict upper limb use in ADL. Methods: Eightytwo patients with a right-hemispheric stroke (RHS) were prospectively included in the study. They were assessed twice in the acute/subacute and in the subacute/chronic phases (mean time interval of 45 days) after stroke. The Catherine Bergego Scale (CBS) was used to quantify the influence of spatial neglect on the ADL. Contralesional upper limb use in the ADL was evaluated with the Lucerne international classification of function, disability and health-based Multidisciplinary Observation Scale. Hand strength was measured using the Jamar, dexterity with the Nine Hole Peg test, and tactile perception using the stereognosis subtest of the Nottingham Sensory Assessment. Cognitive functions were assessed with the Montreal Cognitive Assessment. Results:
\end{abstract}

Regression analyses revealed that spatial neglect is an independent and a significant predictor of upper limb outcome. A CBS score of $\leq 5$ at the time of admission to neurorehabilitation care was highly predictive for good upper limb use in the ADL 45 days later. Conclusions: This study demonstrates that spatial neglect severity, as observed in the ADL, is a significant and an independent predictor of upper limb outcome. Neglect therapy is thus needed to further improve contralesional upper limb use in the ADL in RHS patients.

(c) 2017 S. Karger AG, Basel

\section{Introduction}

Stroke is one of the leading causes of disability in developed countries [1]. To a great extent, disability after stroke is due to impaired motor function, especially in the upper limbs $[2,3]$. In recent years, a large amount of research has been conducted on the recovery and outcome of the upper limbs after stroke $[4,5]$. In most studies, the focus has been set on quantifying impairment according to WHO's international classification of function, disability and health (ICF) [6] by administering arm function tests such as the Fugl-Meyer Assessment [7] and the Action Research Arm test [2]. For instance, Nijland et al. [8] demonstrated that patients showing active shoulder abduction and active finger extension with-

\section{KARGER}

(c) 2017 S. Karger AG, Basel

E-Mail karger@karger.com

www.karger.com/ced
Tim Vanbellingen, $\mathrm{PhD}$

Gerontechnology and Rehabilitation Group

University of Bern

CH-3010 Bern (Switzerland)

E-Mail tim.vanbellingen@dkf.unibe.ch 
in $72 \mathrm{~h}$ after stroke onset have a probability of $98 \%$ to improve by at least 10 points on the action research arm test at 6-month follow-up. Stinear et al. [9] proposed the use of an algorithm called predicting recovery potential, including a clinical motor score, combined with transcranial magnetic stimulation and MRI in order to predict upper limb motor recovery. However, these previous studies did not take into account the functional relevance of this recovery on the level of activity or participation of the ICF [6]. To get a comprehensive picture of functional recovery of the upper limb, it is important to know how the latter is integrated in the activities of daily living (ADL). In fact, the upper limb use does not only depend on preserved motor function but also depends on cognitive and perceptual functions. Among them, the orientation of visual attention is crucial for the execution of the ADL [10]. Disturbed attentional orienting, for instance, in patients with spatial neglect, may have a negative impact on the functional outcome after stroke $[11,12]$. Until now it is not known how the functional recovery of upper limb use in the ADL is influenced by spatial neglect. The aim of this study was thus to investigate whether and how the presence and severity of spatial neglect would predict contralesional upper limb use in the ADL. In order to assess neglect in an ecologically valid setting, the Catherine Bergego Scale (CBS) was used [13]. In order to assess upper limb use in the ADL, we used the Lucerne ICF-based Multidisciplinary Observation Scale (LIMOS) [14, 15]. We hypothesized that the severity of spatial neglect would predict upper limb use in the ADL.

\section{Materials and Methods}

Eighty-two patients (aged between 27 and 90 years, mean 67.87, SD 13.79; 35 women), with a first, right-hemispheric stroke (RHS) participated in this study. Sixty-one patients had an ischemic stroke (A. cerebri media, $n=55$; A. cerebri anterior, $n=3$; A. cerebri posterior, $n=3$ ) and 21 patients had a haemorrhagic stroke (affected regions: frontal, 6/21, parietal, 8/21, temporal 7/21, occipital $1 / 21$, thalamus, $3 / 21$, basal ganglia, $7 / 21$ ). Patients were consecutively enrolled in the study and were all admitted to the Neurocenter, Luzerner Kantonsspital from January 2014 to January 2016, receiving multidisciplinary neurorehabilitation. Patients being motivated and able to actively participate in the neurorehabilitation program were included. Patients who were $100 \%$ bedridden or had additional degenerative or psychiatric diseases were excluded from the study. The study was conducted in accordance with the principles of the latest Declaration of Helsinki (1975) and was approved by the local Ethics Committees of the state of Lucerne. All patients gave their written informed consent prior to participating in this study.

Spatial Neglect Predicts Upper Limb Use in the ADL

\section{Procedures}

The assessments were administered first in the acute/subacute phase (days post stroke: mean 11.3, range $0-30$, hereafter called admission time) and second in the subacute/chronic phase (mean 44.5 , range $22-109$, hereafter called discharge) of stroke. Acute was defined as the condition that exists up to 7 days; subacute was defined as the condition that prevails between days 7 and 30; chronic condition exists $>30$ days post stroke onset [16]. The mean time interval between admission and discharge was 45 days (range 21109). Spatial neglect diagnosis was based on the CBS, which is able to quantify the influence of spatial neglect-related deficits in the ADL [13]. The CBS consists of 10 items, completed by a clinician after observing a patient performing different ADL, such as grooming. Items are scored on a $0-3$ scale, with 0 indicating no neglect and 3 indicating severe neglect (range $0-30$ ). Three levels are distinguished: 1-10 denoted mild neglect; 11-20 denoted moderate neglect; 21-30 denoted severe neglect [13].

Since the primary focus of this study was centered around contralesional upper limb functioning in the ADL, we created a modified upper limb LIMOS score for the left arm [14]. This modified upper limb LIMOS score consists of 5 items, that is, item 5 (lifting up and carrying objects), item 6 (fine hand use), item 7 (hand and arm use), item 13 (washing the upper body), and item 16 (dressing, putting on and taking off clothes in the upper body) of the original LIMOS scale. Every item is rated on a 5-point scale (online suppl. file, see www.karger.com/doi/10.1159/000477500). Isometric hand strength was measured using a JAMAR dynamometer [15]. If patients were able to perform fingers extension, finger and hand function were measured by means of the Nine Hole Peg test [17]. The Montreal Cognitive Assessment (MoCA) was administered in order to screen cognitive abilities [18]. The total maximum possible score of the MoCa is 30 . Stereognosis was assessed by means of the corresponding subscale of the Nottingham Sensory Assessment, which assesses the recognition of 10 objects by using the affected upper limb, scoring performance from 0 (astereognosis) to 2 (normal stereognosis) [19]. The total Nottingham Sensory Assessment score ranges from 0 to 20 .

\section{Statistical Analyses}

For all statistical analyses, the level of significance was set at $p<$ 0.05 (2-tailed). Statistical analyses were performed using PASW for Windows version 24.0 (SPSS Inc., Chicago, IL, USA). Descriptive and clinical characteristics were compared between the 2 measurement time points using paired $t$ tests. Pearson correlation analyses were performed between, on the one hand, demographic variables and behavioural measures at the time of admission and, on the other hand, LIMOS upper limb scores at the time of discharge. To explore which behavioural measures would most strongly predict upper limb performance, a stepwise hierarchical regression analysis was applied with the LIMOS upper limb outcome scores at the time of discharge as the dependent variable, and the behavioural measures as the independent variable. Age was integrated at a first step within the hierarchical regression analysis, followed by motor scores at a second step, cognitive score at a third, tactile perception at a fourth and spatial neglect scores at the fifth and final step. The association between spatial neglect severity and upper limb outcome, controlling for strength and age, was further analyzed by partial correlation analysis. Finally, receiver operating characteristics curves were computed for several cut-off baseline scores in the behavioural measures. Sensitivity, specificity, negative and positive

Cerebrovasc Dis 2017;44:122-127 
Table 1. Admission and discharge scores of behavioural measures in right hemisphere stroke patients $(n=82)$

\begin{tabular}{lllll}
\hline & Admission & Discharge & $p$ values & $\begin{array}{l}\text { Effect size } \\
\text { (Cohen's d) }\end{array}$ \\
\hline CBS & $5.86 \pm 7.49(0-26)$ & $2.89 \pm 5.25(0-24)$ & $<0.0001$ & 0.40 \\
MoCA & $19.27 \pm 6.20(3-30)$ & $21.50 \pm 5.66(6-30)$ & $<0.0001$ & 0.35 \\
9-HPT & $42.05 \pm 19.85(17-100)$ & $32.67 \pm 13.64(16-84)$ & $<0.0001$ & 0.47 \\
Jamar & $19.74 \pm 11.10(0-48)$ & $22.46 \pm 10.32(0-50)$ & $<0.0001$ & 0.24 \\
Stereognosis & $16.02 \pm 5.99(0-22)$ & $18.08 \pm 4.57(0-22)$ & $<0.001$ & 0.34 \\
LIMOS upper limb & $16.01 \pm 5.31(6-25)$ & $19.51 \pm 4.77(9-25)$ & $<0.0001$ & 0.66
\end{tabular}

All values are stated as mean $\pm \mathrm{SD}$ (range).

MoCA, Montreal Cognitive Assessment; CBS, Catherine Bergego Scale; 9-HPT, Nine Hole Peg test values of the left hand; LIMOS, Lucerne ICF-Based Multidisciplinary Observation Scale.

Table 2. Correlations between LIMOS upper limb scores during discharge and demographic and behavioural measures during admission

\begin{tabular}{lcc}
\hline & $\begin{array}{l}\text { LIMOS upper limb } \\
\text { score at discharge }\end{array}$ & $p$ value \\
\hline Age & -0.38 & $<0.001$ \\
CBS & -0.69 & $<0.0001$ \\
Jamar & 0.78 & $<0.0001$ \\
9-HPT & -0.48 & $<0.0001$ \\
MoCA & 0.42 & $<0.0001$ \\
Stereognosis & 0.41 & $<0.0001$ \\
\hline
\end{tabular}

MoCA, Montreal Cognitive Assessment; CBS, Catherine Bergego Scale; 9-HPT, Nine Hole Peg test values of the left hand; LIMOS, Lucerne ICF-Based Multidisciplinary Observation Scale.

Table 3. Results of the hierarchical regression analyses with LIMOS upper limb scores during discharge as the dependent variable

\begin{tabular}{lrlrl}
\hline Scores at admission & \multicolumn{1}{l}{$\beta$} & SE & $t$ value & $p$ value \\
\hline Age & -0.23 & 0.02 & -2.19 & 0.03 \\
Jamar & 0.29 & 0.03 & 2.73 & 0.008 \\
9-HPT & -0.23 & 0.02 & -2.05 & 0.046 \\
MoCA & 0.03 & 0.05 & 0.24 & 0.81 \\
Stereognosis & 0.05 & 0.06 & 0.50 & 0.62 \\
CBS & -0.34 & 0.05 & -3.49 & 0.001 \\
\hline
\end{tabular}

MoCA, Montreal Cognitive Assessment; CBS, Catherine Bergego Scale; 9-HPT, Nine Hole Peg test; LIMOS, Lucerne ICFBased Multidisciplinary Observation Scale.

The regression analysis revealed that the predictive model including all of the above-mentioned factors explained the reason behind $50 \%$ of the variance of upper limb outcome scores during discharge $\left(\mathrm{R}^{2}\right.$ adjusted $\left.=0.50, \mathrm{~F}[6,54]=10.79, p<0.001\right)$. Age accounted for $15 \%$ of the variance of upper limb outcome scores at the time of discharge, and hand strength for $24 \%$ of this variance. An additional $11 \%$ of the variance was explained by spatial neglect. predictive values and the area under the curve (AUC) were calculated in order to identify good upper limb outcome at the time of discharge.

\section{Results}

Spatial neglect symptoms in the ADL, hand strength, fine motor function, cognition, stereognosis and upper limb outcome improved significantly with respect to baseline (Table 1). At the time of admission to the neurorehabilitation unit, $62 \%(51 / 82)$ of the patients demonstrated spatial neglect, whereas this figure decreased to $46 \%$ (38/82) during discharge.

Pearson correlation analyses indicated that the severity of spatial neglect in the ADL at the time of admission was significantly and negatively associated with upper limb outcome during discharge. This was also the case for fine motor function. With respect to hand strength, stereognosis and cognitive functioning, significant positive correlations were found. Finally, age was significantly and negatively correlated with the LIMOS upper limb discharge scores (Table 2).

The hierarchical regression analyses demonstrated significant and strong coefficients for age, hand strength, fine motor function and spatial neglect severity (Tables $3,4)$.

Among these behavioural measures, hand strength and spatial neglect severity were the strongest predictors of upper limb outcome at the time of discharge. The independency of the amount of variance in upper limb outcome explained by spatial neglect was also underlined by the results of the partial correlation analysis, which indicated the presence of a strong association $(r=-0.54, p<$ 0.001 ) between spatial neglect scores at the time of admis- 
Table 4. Intercorrelations of all behavioral variables

\begin{tabular}{|c|c|c|c|c|c|c|c|}
\hline & $\begin{array}{l}\text { LIMOS } \\
\text { upper limb }\end{array}$ & Age & Jamar & 9-HPT & MoCA & Stereognosis & CBS \\
\hline \multicolumn{8}{|c|}{ Correlation coefficients } \\
\hline LIMOS upper limb & 1.00 & -0.38 & 0.78 & -0.48 & 0.42 & 0.41 & -0.69 \\
\hline Age & -0.38 & 1.00 & -0.31 & 0.34 & -0.34 & -0.07 & 0.02 \\
\hline Jamar & 0.78 & -0.31 & 1.00 & -0.39 & 0.34 & 0.20 & -0.23 \\
\hline 9-HPT & -0.48 & 0.34 & -0.39 & 1.00 & -0.42 & -0.30 & 0.18 \\
\hline MoCA & 0.42 & -0.34 & 0.34 & -0.42 & 1.00 & 0.06 & -0.32 \\
\hline Stereognosis & 0.41 & -0.07 & 0.20 & -0.30 & 0.06 & 1.00 & -0.08 \\
\hline CBS & -0.69 & 0.02 & -0.23 & 0.18 & -0.32 & -0.08 & 1.00 \\
\hline \multicolumn{8}{|l|}{ p value } \\
\hline LIMOS upper limb & & 0.00 & 0.00 & 0.00 & 0.00 & 0.00 & 0.00 \\
\hline Age & 0.00 & & 0.01 & 0.00 & 0.00 & 0.29 & 0.45 \\
\hline Jamar & 0.00 & 0.01 & & 0.00 & 0.00 & 0.06 & 0.04 \\
\hline 9-HPT & 0.00 & 0.00 & 0.00 & & 0.00 & 0.01 & 0.09 \\
\hline MoCA & 0.00 & 0.00 & 0.00 & 0.00 & & 0.31 & 0.01 \\
\hline Stereognosis & 0.00 & 0.29 & 0.06 & 0.01 & 0.31 & & 0.27 \\
\hline CBS & 0.00 & 0.45 & 0.04 & 0.09 & 0.01 & 0.27 & \\
\hline
\end{tabular}

MoCA, Montreal Cognitive Assessment; CBS, Catherine Bergego Scale; 9-HPT, Nine Hole Peg test values of the left hand; LIMOS, Lucerne ICF-Based Multidisciplinary Observation Scale.

Table 5. Best predictive cut-off values of Jamar, CBS and 9-HPT for good LIMOS upper limb outcome at discharge

\begin{tabular}{llllll}
\hline Cut-off & Sensitivity (95\% CI) & Specificity (95\% CI) & PPV & NPV & AUC \\
\hline Jamar $\geq 13 \mathrm{~kg}$ & $0.87(0.74-0.94)$ & $0.80(0.61-0.92)$ & $0.88(0.76-0.96)$ & $0.77(0.59-0.90)$ & 0.90 \\
CBS $\leq 5$ & $0.84(0.71-0.93)$ & $0.69(0.50-0.84)$ & $0.81(0.67-0.90)$ & $0.73(0.54-0.88)$ & 0.82 \\
9-HPT $\leq 47 \mathrm{~s}$ & $0.77(0.63-0.87)$ & $0.71(0.42-0.92)$ & $0.91(0.78-0.97)$ & $0.45(0.24-0.68)$ & 0.76 \\
\hline
\end{tabular}

PPV, positive predictive value; NPV, negative predictive value; AUC, area under the curve; CBS, Catherine Bergego Scale; 9-HPT, Nine Hole Peg test.

sion and upper limb outcome at the time of discharge, even when controlling for hand strength and age.

An excellent AUC of 0.90 was found for the Jamar cutoff value of $13 \mathrm{~kg}$. This cut-off value was more sensitive (0.87) than specific (0.80) in predicting good upper limb outcome at the time of discharge. Higher cut-off values $(>13 \mathrm{~kg})$ led to higher sensitivity values $(>0.87)$, but also to lower specificity $(<0.80)$. For the CBS, it was found that for the cut-off value of 5 , the best possible AUC was 0.82 , indicating good diagnostic accuracy, with a high sensitivity of 0.84 and a lower specificity of 0.69 in predicting good upper limb outcome at the time of discharge. For the Nine Hole Peg test, it was found that for a cut-off value of $47 \mathrm{~s}$, the best possible AUC was 0.76 , with a sensitivity of 0.77 and a specificity of 0.71 (Table 5 ).

Spatial Neglect Predicts Upper Limb Use in the ADL
To further assess the independency of the effects of spatial neglect severity from the ones of hand strength, we performed a sub-analysis in a sub-group of patients $(n=$ $25)$ demonstrating spatial neglect $(\mathrm{CBS}>0$ ) but good hand strength at the time of admission (above the cutoff of $13 \mathrm{~kg}$ on the Jamar). The results are presented in Figure 1 .

\section{Discussion}

This study demonstrates that upper limb outcome after stroke not only depends on some degree of preserved hand function [4, 8,9], dexterity [20], or age [21] but also critically on the severity of spatial neglect as observed in 


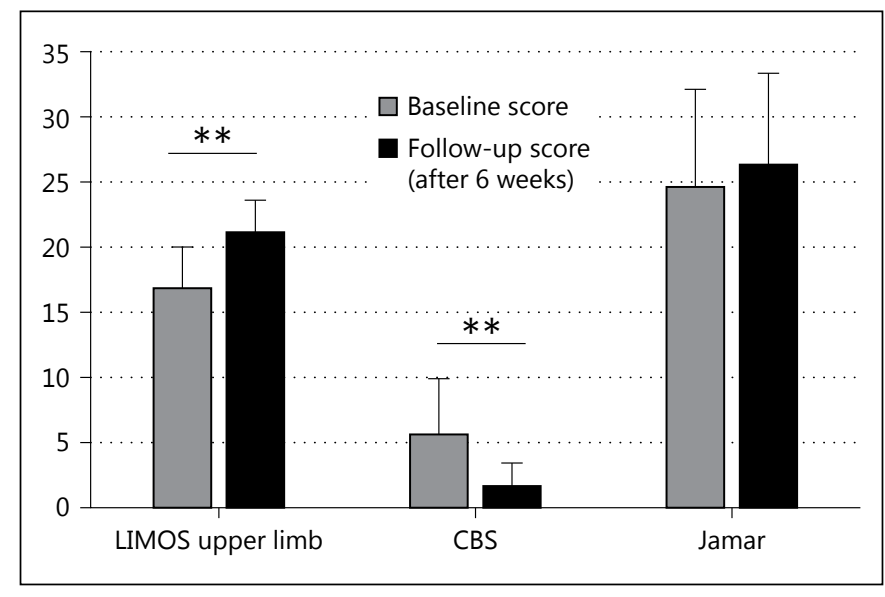

Fig. 1. In neglect patients with hand strength at admission (Jamar $\geq 13 \mathrm{~kg}, n=25)$, LIMOS upper limb scores $(* * p<0.01, \mathrm{~d}=1.32$ ) increased significantly, whereas the CBS scores $\left({ }^{* *} p<0.01, \mathrm{~d}=\right.$ $-0.90)$ decreased significantly.

the ADL. In RHS, a cut-off score of $\leq 5$ in the CBS is sensitive, specific, and highly predictive of good upper limb outcome at neurorehabilitation discharge.

Previous studies investigating neglect severity as a potential predictor of stroke outcome focused on the impairment level but not on the level of activity or participation in social interactions (ICF) [6]. For instance, Nijboer et al. [22, 23] demonstrated that higher neglect severity, as measured by letter cancellation tasks, was associated with a worse outcome. On average, neglect patients were more impaired in self-care, transfer and locomotion compared to non-neglect patients [23]. In addition, for the upper limb, a longitudinal association was found between letter cancellation impairment and FuglMeyer Assessment scores [22]. These studies thus provided some insight into a possible negative impact of spatial neglect on upper limb outcome. However, the ascertainment of a direct relationship between the severity of neglect and the use of the upper limb in the ADL was still lacking.

In this study, we measured spatial neglect severity at the level of the ADL, as recommended by the ICF [6]. We used the CBS, which is more sensitive than paper-pencil tasks [13]. In fact, dissociations in paper-pencil task results are well known: patients may perform cancellation tasks normally but be severely impaired while performing line-bisection tasks and vice versa $[24,25]$. In contrast to other tools, an additional advantage of the CBS is that it is possible to observe spontaneous behaviour during the ADL, while at the same time not exposing the patients to a test kind of a situation. In this study, we show for the first time that spatial neglect, as observed in the $\mathrm{ADL}$, is an independent predictor of future functional upper limb use. Furthermore, we provide an optimum cut-off value for the CBS (i.e., $\leq 5)$ for good upper limb outcome at 6 weeks. The fact that we obtained neither $100 \%$ sensitivity nor $100 \%$ specificity for this cut-off value implies that some RHS patients still achieved a poor upper limb outcome despite having low CBS scores. On the other hand, it is known that some patients with neglect may lose motor spontaneity in their left hand despite having normal strength [26]. Our sub-analysis in the sub-group of neglect patients showing good hand strength at the time of admission demonstrates that a decrease in spatial neglect severity coincided with a more effective upper limb use in the ADL, possibly contributing to a more effective neurological recovery.

The strongest predictor for a good upper limb outcome was represented by preserved hand strength $(\geq 13$ $\mathrm{kg}$ on the Jamar). This underlines the importance of corticomotor tract integrity in order to achieve an optimal motor outcome $[9,27,28]$. Furthermore, in line with the literature, we found that younger age [21], good dexterity [20] and good tactile perception [29] were related to good outcome. In contrast to previous studies $[8,9,22]$, in our study, we included stroke patients with cognitive deficits. This is more representative of the population of RHS patients actually admitted to neurorehabilitation centres, who very often present with cognitive impairments [18]. Our results show that lower MoCA scores at the time of admission were related to worse upper limb outcome at the time of discharge. This finding is very relevant, since existing predictive algorithms have so far only focused on the predictive value of motor scores $[8,9]$. Our study thus extends this knowledge and suggests that future investigations should ideally also include a cognitive score such as the CBS in algorithms aiming at predicting functional recovery.

A possible limitation of this study is that we did not assess our patients at a later follow-up time point (e.g., 3 months). Therefore, the predictive role of spatial neglect for upper limb outcome on the long term still needs to be clarified. However, the focus of this study was set on an earlier time period, since the mean inpatient neurorehabilitation stay in Europe is about 6 weeks [30]. In addition, it has been shown that spontaneous recovery is most pronounced over the first month after stroke [31]. Future studies could combine the predictive role of paper-pencil tests and ADL-based neglect scores. For this purpose, a comprehensive neglect battery, assessing body, peri- and extra-personal aspects, similar to the CBS, would be re- 
quired. Furthermore, the impact of anosognosia of neglect on upper limb use could also be evaluated, since it may affect ADL in general [32].

\section{Conclusions}

To conclude, this study provides evidence that spatial neglect severity, as observed in the ADL, is an independent predictor of functional upper limb outcome. We also provide an optimal cut-off value for the CBS (i.e., $\leq 5$ ), having a good predictive validity for good upper limb outcome at 6 weeks after stroke. This suggests that in ad- dition to conventional physiotherapy and occupational therapy, neglect therapy is quite important to improve upper limb outcome in RHS patients.

\section{Acknowledgments}

This study was supported by 2 grants from the Swiss National Science Foundation (320030_169789, 32003B_155954).

\section{Disclosure Statement}

The authors have no conflicts of interest to declare.

\section{References}

1 World Health Organization: The Global Burden of Disease: 2004 Update. Geneva, World Health Organization, 2004.

2 Veerbeek JM, Kwakkel G, van Wegen EE, Ket JC, Heymans MW: Early prediction of outcome of activities of daily living after stroke: a systematic review. Stroke 2011;42:1482-1488.

3 Nakayama H, Jørgensen HS, Raaschou HO, Olsen TS: Recovery of upper extremity function in stroke patients: the Copenhagen Stroke Study. Arch Phys Med Rehabil 1994; 75:394-398.

4 Byblow WD, Stinear CM, Barber PA, Petoe MA, Ackerley SJ: Proportional recovery after stroke depends on corticomotor integrity. Ann Neurol 2015;78:848-859.

5 Winters C, van Wegen EE, Daffertshofer A, Kwakkel G: Generalizability of the maximum proportional recovery rule to visuospatial neglect early poststroke. Neurorehabil Neural Repair 2017;31:334-342.

6 World Health Organization: International Classification of Functioning, Disability and Health (ICF), 2001.

7 Fugl-Meyer AR, Jääskö L, Leyman I, Olsson S, Steglind S: The post-stroke hemiplegic patient. 1. A method for evaluation of physical performance. Scand J Rehabil Med 1975;7: 13-31.

8 Nijland RH, van Wegen EE, Harmeling-van der Wel BC, Kwakkel G; EPOS Investigators: Presence of finger extension and shoulder abduction within 72 hours after stroke predicts functional recovery: early prediction of functional outcome after stroke: the EPOS cohort study. Stroke 2010;41:745-750.

9 Stinear CM, Barber PA, Petoe M, Anwar S, Byblow WD: The PREP algorithm predicts potential for upper limb recovery after stroke. Brain 2012;135(pt 8):2527-2535.

10 Erel H, Levy DA: Orienting of visual attention in aging. Neurosci Biobehav Rev 2016;69: 357-380.

11 Di Monaco M, Schintu S, Dotta M, Barba S, Tappero R, Gindri P: Severity of unilateral spatial neglect is an independent predictor of functional outcome after acute inpatient rehabilitation in individuals with right hemispheric stroke. Arch Phys Med Rehabil 2011; 92:1250-1256.

12 Gialanella B, Ferlucci C: Functional outcome after stroke in patients with aphasia and neglect: assessment by the motor and cognitive functional independence measure instrument. Cerebrovasc Dis 2010;30:440-447.

13 Azouvi P: The ecological assessment of unilateral neglect. Ann Phys Rehabil Med 2016; pii:S1877-0657(16)00003-8.

14 Ottiger B, Vanbellingen T, Gabriel C, Huberle E, Koenig-Bruhin M, Pflugshaupt T, et al: Validation of the new Lucerne ICF based Multidisciplinary Observation Scale (LIMOS) for stroke patients. PLoS One 2015;10:e130925.

15 Vanbellingen T, Ottiger B, Pflugshaupt T, Mehrholz J, Bohlhalter S, Nef T, et al: The responsiveness of the Lucerne ICF-based Multidisciplinary Observation Scale: a comparison with the functional independence measure and the Barthel index. Front Neurol 2016;7:152.

16 Hankey GJ: Stroke. Lancet 2017;389:641-654.

17 Chen HM, Chen CC, Hsueh IP, Huang SL, Hsieh CL: Test-retest reproducibility and smallest real difference of 5 hand function tests in patients with stroke. Neurorehabil Neural Repair 2009;23:435-440.

18 Chiti G, Pantoni L: Use of Montreal Cognitive Assessment in patients with stroke. Stroke 2014;45:3135-3140.

19 Lincoln NB, Jackson JM, Adams SA: Reliability and revision of the Nottingham sensory assessment for stroke patients. Physiotherapy 1998;84:358-365.

20 Wagner JM, Lang CE, Sahrmann SA, et al: Sensorimotor impairments and reaching performance in subjects with poststroke hemiparesis during the first few months of recovery. Phys Ther 2007;87:751-765.

21 Bagg S, Pombo AP, Hopman W: Effect of age on functional outcomes after stroke rehabilitation. Stroke 2002;33:179-185.
22 Nijboer T, van de Port I, Schepers V, Post M, Visser-Meily A: Predicting functional outcome after stroke: the influence of neglect on basic activities in daily living. Front Hum Neurosci 2013;7:182.

23 Nijboer TC, Kollen BJ, Kwakkel G: The impact of recovery of visuo-spatial neglect on motor recovery of the upper paretic limb after stroke. PLoS One 2014;9:e100584.

24 Verdon V, Schwartz S, Lovblad KO, Hauert CA, Vuilleumier P: Neuroanatomy of hemispatial neglect and its functional components: a study using voxel-based lesion-symptom mapping. Brain 2010;133(pt 3):880-894.

25 Bowen A, Lincoln NB: Cognitive rehabilitation for spatial neglect following stroke. Cochrane Database Syst Rev 2007;2:CD003586.

26 Laplane D, Degos JD: Motor neglect. J Neurol Neurosurg Psychiatry 1983;46:152-158.

27 Feng W, Wang J, Chhatbar PY, Doughty C, Landsittel D, Lioutas VA, et al: Corticospinal tract lesion load: an imaging biomarker for stroke motor outcomes. Ann Neurol 2015;78: 860-870.

28 Carmichael ST: Emergent properties of neural repair: elemental biology to therapeutic concepts. Ann Neurol 2016;79:895-906.

29 Wu CY, Chuang IC, Ma HI, Lin KC, Chen CL: Validity and responsiveness of the revised Nottingham sensation assessment for outcome evaluation in stroke rehabilitation. Am J Occup Ther 2016;70:7002290040p1-8.

30 Rollnik JD, Janosch U: Current trends in the length of stay in neurological early rehabilitation. Dtsch Arztebl Int 2010;107:286-292.

31 Duncan PW, Goldstein LB, Matchar D, Divine GW, Feussner J: Measurement of motor recovery after stroke. Outcome assessment and sample size requirements. Stroke 1992; 23:1084-1089.

32 Vossel S, Weiss PH, Eschenbeck P, Fink GR: Anosognosia, neglect, extinction and lesion site predict impairment of daily living after right-hemispheric stroke. Cortex 2013;49: 1782-1789. 